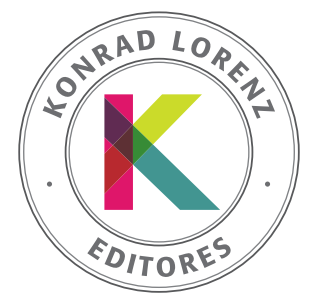

SUMA PSICOLÓGICA

http://editorial.konradlorenz.edu.co/suma-psicologica.html

\title{
Preditores do envolvimento paterno numa amostra de famílias portuguesas. 0 papel das crenças parentais
}

\author{
Lígia Monteiro $^{\mathrm{a}, *}$, Nuno Torres $^{\mathrm{b}}$ y Fernando Salinas-Quiroz ${ }^{\mathrm{c}}$
}

\begin{abstract}
${ }^{a}$ Departamento de Psicologia Social e das Organizações do ISCTE - Instituto Universitário de Lisboa, CIS-IUL, Lisboa, Portugal
${ }^{b}$ ISPA-Instituto Universitário, e Investigador em Psicologia do Desenvolvimento financiado pela Fundação Para a Ciência e Tecnologia, William James Research Center, Lisboa, Portugal

' Universidad Pedagógica Nacional, Ajusco, Ciudad de México, México
\end{abstract}

Recibido el 16 de mayo de 2019; aceptado el 6 de agosto de 2019

\section{PALAVRAS-CHAVE}

Envolvimento paterno, crenças sobre o papel do pai, gatekeeping

\begin{abstract}
Resumo 0 presente estudo visou analisar preditores do envolvimento paterno em atividades do dia-a-dia relacionadas com a criança, considerando a perspetiva do pai (crenças sobre o papel do pai) e testando o possível papel regulador da mãe (via crenças maternas). Foram controladas variáveis sociodemográficas dos cuidadores (e.g., habilitações) e da criança (e.g., sexo). Participaram no estudo 241 famílias nucleares (pai, mãe e criança-alvo). Análises de regressão múltipla hierárquica indicam que apenas os modelos relativos aos cuidados são significativos, e que os preditores são distintos consoante os domínios analisados. Obteve-se, ainda, um efeito de interação entre as crenças maternas e o sexo da criança, indicando o potencial papel regulatório da mãe no modo como os pais estão envolvidos nos cuidados diretos às raparigas. Estes resultados colocam questões importantes para o trabalho com as famílias no sentido de promover um maior envolvimento positivo do pai.

(c) 2019 Fundación Universitaria Konrad Lorenz. Este é um artigo de acesso aberto sob a licença CC BY-NC-ND (http://creativecommons.org/licenses/bync-nd/4.0/).
\end{abstract}

\section{Predictors of Father's Involvement in a Sample of Portuguese families. The Role of} Parenting Beliefs

Abstract The present study aimed to analyze predictors of father's involvement in day-to-day activities related to the child, from his perspective (beliefs about the role of the father), but also testing the regulatory role of the mother (maternal beliefs). Parents (e.g., education) and child's (e.g., sex) sociodemographic variables were controlled. Participants were 241 Portuguese bi-parental families (father, mother and target-child). Multiple hierarquical regression analyses showed that only for care the models reached significance, and that the predictors of father's involvement vary according to the domains analyzed. An interaction effect was found between mother's beliefs and child sex, suggesting that mothers have a role in the way fathers are involved in direct care with girls. These results foster important questions for interventions with families in order to promote father's positive involvement.

(c) 2019 Fundación Universitaria Konrad Lorenz. This is an open access article under the CC BY-NCND license (http://creativecommons.org/licenses/bync-nd/4.0/).

\footnotetext{
* Autor para correspondencia.

Correo electrónico: ligia.monteiro@iscte-iul.pt
} 
Os papéis de género assumidos por mulheres e homens, no contexto da sociedade portuguesa, têm sofrido enormes alterações enquadradas por mudanças macrossociais ao nível económico e político, em particular pelas políticas publicas relativas ao emprego e à família. De uma organização social e familiar onde o homem era tido como o pilar económico e de autoridade, e a mulher como esposa, organizadora das tarefas domésticas e natural cuidadora da criança, passou-se a uma organização que procura promover a igualdade de ambos no acesso ao mercado de trabalho, e uma partilha do poder e responsabilidades na família (Wall et al., 2016). Embora a percentagem de mulheres no mercado de trabalho com crianças pequenas seja das mais elevadas da União Europeia (Torres et al., 2018), continua a existir uma disparidade grande no número de horas que homens e mulheres despendem em trabalho doméstico. Porém, têm-se verificado mudanças ao nível dos cuidados à criança, sendo comum a perceção de que quando o pai não participa dos mesmos, isso terá implicações negativas para o seu bem-estar (Wall et al., 2016). Na realidade, esta perceção social vai ao encontro de diversos estudos na área do desenvolvimento humano que indicam que um maior envolvimento positivo do pai se encontra associado com bons resultados da criança nos domínios socio-emocional, cognitivo ou físico (Cabrera, Tamis-LeMonda, Bradley, Hofferth, \& Lamb, 2000; Lamb \& Lewis, 2010), bem como com o próprio desenvolvimento do adulto (Palkotivz, 2002). Estas mudanças parecem ser, contudo, mais lentas e complexas do que por vezes as crenças populares poderiam indicar (Pleck \& Masciadrelli, 2004), sendo que o contexto cultural e as condições sociais parecem ter impacto no modo como os cuidadores e, em particular, o homem define e vivência a parentalidade (Marsiglio, Amato, Lamb, \& Day 2000).

De acordo com diversos autores (ver Parke, 2000), quando falamos do pai e do seu envolvimento na vida da criança, há que ter em consideração que este pode variar em função do tipo de atividades a realizar (e.g., cuidados, brincadeira ou de suporte financeiro), diferenciando-se contextos e tipos de interação, numa visão de que este é um fenómeno complexo e multidimensional. Modo geral, na literatura as interações mãe/criança são marcadas pelos cuidados, dando-se menor saliência ao contexto de brincadeira, enquanto o pai é, essencialmente, caracterizado como parceiro de brincadeira estimulante, desafiante e de natureza mais física, que suporta a exploração ativa do meio (Grossmann et al., 2002). No contexto português, estudos com famílias nucleares (e.g., Monteiro, Fernandes, Torres, \& Santos, 2017; Torres, Veríssimo, Monteiro, Ribeiro, \& Santos, 2014), apontam para uma menor participação relativa do pai face à mãe nas atividades de cuidados (e.g., dar banho ou vestir) ou de gestão (e.g., comprar roupas), enquanto nos domínios da brincadeira (e.g., fazer jogos de tabuleiro ou brincadeiras com características mais físicas), lazer (e.g., ida ao parque), ou ensino/disciplina (ensinar competências ou estabelecer regras) parece haver uma participação tendencialmente igualitária. No entanto, outros estudos indicam que as mães se encontram mais envolvidas tantos nos cuidados como na brincadeira (e.g., Fuertes, Faria, Beeghly, \& Lopes-dos-Santos, 2016).

No sentido de melhor compreender a variabilidade do papel do pai e o seu envolvimento com a criança, há que considerar múltiplos determinantes (Lamb \& Tamis-LeMonda, 2004; Parke, 1996), entre os quais as crenças parentais.
Estas são definidas como sendo promotoras de, e como moldando os comportamentos dos cuidadores ajudando-os a organizar a parentalidade (Bornstein, 2002). Mais do que as atitudes gerais sobre o género, serão as crenças específicas face ao papel de pai (e a sua importância), que parecem ser mais relevantes, dado ser menos normativo para o homem estar envolvido nos cuidados diários aos filhos (Beitel \& Parke, 1998, Palkovitz, 2002; Parke, 1996). Considera-se, assim, que estas crenças poderão ser uma influência proximal e de maior impacto nos comportamentos e práticas do que os constrangimentos contextuais (Freeman, Newland, \& Coyl-Shepherd, 2008). Pais com crenças mais positivas e modernas sobre o seu papel, competências nos cuidados e impacto no desenvolvimento da criança, tendem a estar mais envolvidos, comparativamente com pais com crenças mais tradicionais (Pleck \& Masciadrelli, 2004). As crenças sobre este "novo pai" cuidador e afetivo, que vai para além do disciplinador ou provedor da família, estão associadas a diferentes domínios do envolvimento, inclusivamente com uma maior participação nos cuidados e na interação com a criança (Favez, Tissot, Frascarolo, Stiefel, \& Despland, 2016; Pleck \& Masciadrelli, 2004), ou mesmo com o tempo passado com esta enquanto cuidador primário (Jacobs \& Kelly, 2006). Buckley e Schoppe-Sullivan (2010) verificaram que quando os cuidadores tinham crenças menos tradicionais, os pais encontravam-se mais envolvidos nos cuidados, mas não na brincadeira.

Apesar de uma visão do pai como sendo autodeterminado no seu envolvimento com a criança, há que considerar que este está inserido numa rede complexa de relações, nomeadamente, na família. Assim, o papel que a mulher poderá ter no modo como o homem vivencia a parentalidade deve ser considerado (Marsiglio et al., 2000), dada a centralidade que esta sempre assumiu na educação da criança (Wall et al., 2016). Segundo Allen e Hawkins (1999) as crenças maternas acerca da parentalidade, em particular sobre o papel do pai, bem como os seus comportamentos podem levar a que funcione como gatekeeper, desencorajando ou inibindo um esforço colaborativo com o pai, e restringindo deste modo o seu envolvimento (Fagan \& Barnett, 2003; McBride et al., 2005). Contudo, focar apenas o papel inibidor da mãe será uma perspetiva redutora do fenómeno, devendo-se considerar que esta poderá ter um papel "regulador" podendo restringir/bloquear, mas também facilitar/incentivar o envolvimento do pai no dia-a-dia da criança (Schoppe-Sullivan, Brown, Cannon, Magelsdorf, \& Sokolowski, 2008).

As crenças maternas sobre o papel e importância do pai para o desenvolvimento da criança são um possível mecanismo da função regulatória da mãe no tipo e níveis de envolvimento paterno (Fagan \& Barnett, 2003; McBride et al., 2005). Beitel e Parke (1998) verificaram que tanto as crenças do pai, como da mãe se encontravam associadas com o envolvimento paterno, bem como com os seus níveis de estimulação na interação com a criança. Schoppe-Sullivan et al. (2008) indicam que mães com crenças mais progressistas reportavam maior envolvimento do pai. Numa amostra portuguesa, Monteiro, Torres, Branco, Pessoa e Costa e Santos (2015) identificaram as crenças modernas das mães como o único preditor do envolvimento do pai nos cuidados diretos, enquanto as crenças de ambos os cuidadores previam o envolvimento na brincadeira. Mães com uma visão mais tradicional dos papéis de género poderão percecionar um 
maior envolvimento do pai como o não respeitar de papéis e fronteiras; por outro lado, mães com expectativas mais igualitárias poderão interpretar uma menor participação como distanciamento emocional (Favez et al., 2016). No entanto, Pleck e Masciadrelli (2004) consideram que embora exista alguma evidência do fenómeno de gatekeeping, esta não parece ser robusta.

Os recursos e oportunidades económicas e sociais poderão ter impacto nas atitudes parentais, assim como na natureza e quantidade do envolvimento do pai, pelo que as habilitações académicas e a situação profissional dos progenitores são preditores importantes a analisar. Em amostras portuguesas verificou-se que pais com habilitações mais elevadas participavam mais nas atividades de cuidados (e.g., Monteiro et al., 2010), ensino/disciplina e brincadeira/lazer (e.g., Monteiro et al., 2017). Pais com maior escolaridade poderão ter mais recursos, competências e informação sobre as necessidades e características desenvolvimentais da criança, sentindo-se mais seguros e motivados para interagirem com e participarem dos seus cuidados (Bailey, 1994). Contudo, outros estudos não reportam associações entre habilitações e envolvimento (Afonso, Veríssimo, Fernandes, Borges, \& Monteiro, 2011).

0 emprego das figuras parentais pode colocar diversos desafios e constrangimentos de tempo, com implicações no modo como as responsabilidades parentais são assumidas e partilhadas. Tal é, particularmente, relevante na sociedade portuguesa onde a maioria das famílias são caracterizadas pelo duplo-emprego (Torres et al., 2018), passando a ser socialmente expectável uma maior participação do homem no contexto familiar, como uma via de se atingir a igualdade de género. Diversos estudos indicam que o envolvimento do pai está associado com a mãe trabalhar ou não, e com as horas que ambos os cuidadores trabalham, embora varie em função de se analisar a proporção do tempo total passado com a criança ou do tempo em absoluto (Pleck \& Masciadrelli, 2004). Em famílias portuguesas de duploemprego são quase sempre as mães a realizar as atividades de cuidados e de gestão/organização, surgindo o pai como uma ajuda complementar (e.g., Monteiro et al., 2010, Fuertes et al., 2016). Alguns estudos reportam, no entanto, que as horas de trabalho das mães estão associadas com o maior envolvimento relativo do pai nos cuidados (Schoppe-Sullivan et al., 2008). Dados do estudo NICHD Early Child Care Research Network (2000) indicam que os pais assumiam mais responsabilidades quando trabalhavam menos horas e as mães tinham horários mais longos, sendo que quando as mães trabalhavam a tempo inteiro, os pais participavam mais independentemente do seu tipo de crenças. Por outro lado, o número de horas de trabalho do pai aparece, em alguns estudos, negativamente associado com o seu envolvimento nos cuidados e socialização (Jacob \& Kelley, 2006; Monteiro et al., 2017).

Relativamente às características das crianças que poderão ter impacto no envolvimento do pai, há que considerar a idade, dado que a diferentes períodos de desenvolvimento estão associadas competências de interação e autonomia bastante destintas. Os anos pré-escolares implicam ganhos importantes ao nível linguístico, cognitivo e socio-emocional tornando a criança um parceiro mais atrativo e competente, e representando um pico nos níveis de interações pai/criança, em particular, no contexto de brincadeira (Lamb \& Lewis, 2010; Parke, 1996). Relativamente ao sexo,
Pleck e Masciadrelli (2004) salientam que esta variável parece exercer, atualmente, menor influência sobre o envolvimento, comparativamente com décadas anteriores. Contudo, quando são reportadas diferenças, são no sentido de os pais passarem mais tempo e estarem mais envolvidos com os rapazes, podendo ser esta uma via de socialização em termos dos papéis de género característicos do grupo sociocultural de pertença. Em amostras portuguesas os resultados são mistos, verificando-se que os pais estão igualmente envolvidos com raparigas e rapazes (e.g., Monteiro et al., 2017; Torres et al., 2014), enquanto noutras os pais encontram-se mais envolvidos com os rapazes em atividades de cuidados e/ou brincadeira (e.g., Lima, 2005; Monteiro et al., 2010).

Segundo Cabrera, Volling e Barr (2018), apesar do aumento do número de estudos que integra o pai nas suas análises, este continua a ser em muitos casos "um contributo silencioso" para o desenvolvimento da criança, ou quando incluído é-o através da perspetiva da mãe. Por outro lado, para uma melhor compreensão do pai há que analisar fatores que promovam ou inibam o seu envolvimento, não caindo no erro de generalizar, em demasia, os resultados obtidos em amostras com características étnicas, culturais ou económicas distintas. 0 presente estudo visa ser um contributo nesse sentido, ao analisar o envolvimento relativo do pai, em famílias nucleares portuguesas, ao nível da sua participação em atividades de cuidados, brincadeira e ensino/disciplina que implicam interação direta com a criança, podendo assim ser potencializadoras do seu desenvolvimento (Pleck \& Masciadrelli, 2004), bem como em atividade de gestão/organização (cuidados indiretos) que contribuem para o seu bem-estar. Saliente-se que o envolvimento relativo do pai é analisado com base na sua própria perspetiva, no entanto, dado alguns autores (e.g., Mikelson, 2008) considerarem que os pais sobrestimam a sua participação é, também, pedido às mães que descrevam o envolvimento do pai, permitindo assim testar o acordo entre as respostas dos cuidadores.

Visa-se, ainda, compreender se o envolvimento relativo do pai, em domínios de cuidados e socialização, é explicado pelas suas crenças sobre o papel do pai, ou se é regulado pelas crenças maternas, aqui consideradas como um potencial mecanismo de gatekeeping (McBride et al., 2005). Espera-se que as crenças modernas sejam, particularmente, relevantes no domínio dos cuidados culturalmente associados com as "habilidades naturais da mulher" e o seu papel na família. Esta função reguladora é, ainda, explorada considerando o sexo da criança. As habilitações académicas (número de anos de escolaridade) e o emprego (número de horas semanais) de ambos os cuidadores são considerados como variáveis que podem facilitar/dificultar o maior envolvimento do pai.

\section{Método}

\section{Participantes}

Participaram 241 famílias nucleares portuguesas (pai, mãe e criança-alvo). As mães tinham idades entre os $22 \mathrm{e}$ 50 anos $(M=35.7 ; D P=4.8)$ e os pais entre 19 e 59 anos $(M=37.8 ; D P=5.9)$. As habilitações académicas de mães e pais variavam entre o $1^{\circ}$ ciclo do ensino básico e o ensino superior $\left(M_{\text {mãe }}=14.3\right.$ anos de escolaridade; $D P_{\text {mãe }}=3.5$; 
$M_{\text {pai }}=12.8$ anos; $\left.D P_{\text {pai }}=3.6\right)$. Setenta e nove por cento das mães e $89 \%$ dos pais trabalhavam, as mães em média 38.34 horas semanais $(D P=6.37)$ e os pais 41.5 horas $(D P=6.49)$. As crianças tinham entre 26.5 e 76.9 meses de idade $(M=$ 54.8; $\mathrm{DP}=10.8$ ), sendo $51 \%$ do sexo feminino. 0 rendimento familiar era em média de $1.868 €$, DP $=854 €)$.

\section{Instrumentos/procedimento}

Este estudo foi conduzido de acordo com os padrões éticos da American Psychological Association, e aprovado pela comissão de ética do ISCTE - Instituto Universitário de Lisboa, fazendo parte de um projeto mais amplo sobre as implicações do envolvimento do pai no desenvolvimento socio-emocional da criança. As famílias foram recrutadas através de Jardins-de-Infância localizados no distrito de Lisboa. Todos os participantes assinaram um consentimento informado antes da recolha dos dados, tendo os questionários sido preenchidos de modo independente por mãe e pai e com referência apenas à criança-alvo (que participava no projeto mais amplo).

A Escala de envolvimento parental: participação em atividades de cuidados e de socialização (Monteiro, Veríssimo, \& Pessoa e Costa, 2008) analisa a percepção que o pai (ou mãe) tem acerca da sua participação relativa face à mãe (ou ao pai), na organização e realização de diferentes atividades relacionadas com a criança, e que ocorrem no dia a dia das famílias. Os 26 itens estão organizados em cinco dimensões: 1) cuidados diretos, relacionados com tarefas de cuidados à criança que implicam interação direta (e.g., quem dá as refeições à criança); 2) cuidados indiretos, que remetem para tarefas de organização/planeamento das necessidades e rotinas da criança, não implicando, necessariamente, interação (e.g., quem costuma ir às reuniões de escola do seu filho(a); 3) ensino/disciplina, relacionados com o ensino de competências e o estabelecimento e cumprimento de regras (e.g., quem ensina à criança novas competências (e.g., as cores, jogar um jogo, ou comer à mesa); 4) brincadeira, relacionados com brincadeiras mais tranquilas/mediadas por objetos ou mais físicas/ativas (e.g., quem é que faz jogos mais físicos com o seu filho (ex. jogar à bola, andar às cavalitas, rolar no chão, etc.); 5) lazer no exterior, remete para atividades realizadas com a criança fora de casa (e.g., quem leva o seu filho ao parque infantil). 0 envolvimento é avaliado numa perspetiva relativa, ou seja, como é que as atividades são geridas ou partilhadas em relação à outra figura parental. Foi pedido ao pai e à mãe que respondessem a cada item numa escala de cinco pontos: sempre a mãe (1); quase sempre a mãe (2); tanto a mãe como o pai (3); quase sempre o pai; sempre o pai (5).

Com base nos coeficientes de correlação intra-classe verificou-se que as dimensões: cuidados diretos (.80), cuidados indiretos (.83), ensino/disciplina (.54) e brincadeira (.70) apresentavam valores altos ou muito altos de concordância no casal. Assim, foi calculado um valor compósito (através da média aritmética) das respostas de mães e pais, utilizado nas análises seguintes. Os alfas de Cronbach alcançaram valores aceitáveis nos cuidados diretos (.71), cuidados indiretos (.68), ensino/disciplina (.72), e brincadeira (.66). O lazer no exterior apresentou um alfa de .60 pelo que não foi utilizado.

As crenças sobre o papel do pai foram analisadas com base no questionário What is a Father? (Schoppe, 2001;
Monteiro et al., 2015). Este é composto por 15 itens que remetem para crenças tradicionais e não tradicionais sobre o papel do pai. Os cuidadores responderam numa escala de cinco pontos que varia entre (1) Discordo fortemente e (5) Concordo fortemente. Os itens tradicionais são invertidos, de modo a que valores mais elevados são indicativos de crenças não tradicionais. Neste estudo utilizou-se uma dimensão denominada de crenças modernas sobre o papel do pai, na perspetiva do pai $(\alpha=.67)$ e da mãe $(\alpha=.70)$.

As mães preencheram um questionário sobre dados sociodemográficos de ambos os cuidadores (e.g., habilitações académicas, estado civil), da criança (e.g., idade, sexo), e da família (e.g., rendimento).

\section{Plano de Análises}

Em primeiro lugar calcularam-se as estatísticas descritivas das variáveis do envolvimento relativo do pai e das crenças modernas sobre o papel do pai. Seguidamente analisaram-se as: (1) diferenças entre o pai e a mãe ao nível das suas crenças através de uma análise de variância de medidas repetidas; (2) associações bivariadas e diferenças de médias entre variáveis sociodemográficas, e o envolvimento e crenças parentais através de correlações de Pearson para as variáveis contínuas, e de testes $t$ para as variáveis dicotómicas (sexo da criança); (3) associações bivariadas entre envolvimento e as crenças parentais através de correlações de Pearson. Finalmente, realizaram-se modelos de regressão múltipla hierárquica em que as dimensões do envolvimento foram incluídas como variáveis dependentes, e as variáveis sociodemográficas e as crenças parentais foram incluídas como preditores. As análises foram realizadas com o pacote IBM SPSS 21.0.

\section{Resultados}

Os resultados das estatísticas descritivas (Tabela 1) indicam que os cuidados diretos e indiretos são em média quase sempre realizados pela mãe, enquanto as atividades de ensino/disciplina e brincadeira são tendencialmente partilhadas por ambos os cuidadores, com particular saliência para o contexto de brincadeira.

Tabela 1. Estatísticas descritivas do envolvimento relativo do pai e das crenças sobre o papel do pai $(\mathrm{N}=241)$

\begin{tabular}{lcccc}
\hline & Mínimo & Máximo & Média & $\begin{array}{c}\text { Desvio } \\
\text { padrão }\end{array}$ \\
\hline $\begin{array}{l}\text { Envolvimento cuidados } \\
\text { diretos }\end{array}$ & 1.11 & 3.41 & 2.47 & .47 \\
\hline $\begin{array}{l}\text { Envolvimento cuidados } \\
\text { indiretos }\end{array}$ & 1.21 & 3.64 & 2.39 & .41 \\
\hline $\begin{array}{l}\text { Envolvimento ensino/ } \\
\text { disciplina }\end{array}$ & 1.41 & 3.61 & 2.84 & .31 \\
\hline $\begin{array}{l}\text { Envolvimento brinca- } \\
\text { deira }\end{array}$ & 1.81 & 3.81 & 3.03 & .35 \\
\hline Crenças modernas pai & 3.33 & 5.00 & 4.36 & .39 \\
\hline Crenças modernas mãe & 3.33 & 5.00 & 4.34 & .39 \\
\hline
\end{tabular}

Relativamente às crenças sobre o papel do pai, ambos os cuidadores apresentam, em média, crenças progressistas. A 
realização de uma análise de variância de medidas repetidas indica que não existem diferenças significativas entre os valores das crenças de pais e mães $(\mathrm{F}[1,240]=.789 ; p=.38)$.

As associações entre a informação sociodemográfica (medida em variáveis contínuas) e as variáveis relativas às crenças modernas e envolvimento relativo do pai foram analisadas através de correlações de Pearson. Relativamente ao pai, encontrou-se uma associação significativa e negativa entre a idade e as suas crenças $(r=-.18 ; p=.041)$, e positiva entre as suas habilitações académicas e as crença da mãe $(r=.26 ; p<.001)$, e as suas habilitações e o envolvimento nos cuidados diretos $(r=.16, p=.018)$, indiretos $(r=.28 ; p<.001)$, ensino/disciplina $(r=.22 ; p=.001) \mathrm{e}$ brincadeira $(r=.18 ; p=.027)$. As horas de trabalho do pai estão negativamente associadas com as crenças maternas $(r=-.14 ; p=.034)$. Para a mãe, encontraram-se associações positivas e significativas entre as suas habilitações académicas e o envolvimento do pai nos cuidados diretos $(r=.12$; $p=.045)$, indiretos $(r=.15 ; p=.042)$ e brincadeira $(r=.19$; $p=.029)$; e entre as suas habilitações e as suas crenças $(r=.25 ; p<.001)$. Verificou-se, ainda, uma associação positiva e significativa entre número de horas de trabalho da mãe e o envolvimento do pai nos cuidados diretos $(r=.20$; $p=.027)$. Não existem associações significativas com a idade da criança $(p>.05)$. No sentido de testar a existência de diferenças em função do sexo da criança para as crenças parentais e envolvimento do pai realizaram-se testes $t$; nenhum atingiu significância estatística $(p>.05)$.

Testaram-se, de seguida, as associações entre as dimensões do envolvimento relativo do pai e as crenças parentais (Tabela 2).

Tabela 2. Correlações de Pearson entre o envolvimento relativo do pai e as crenças sobre o papel do pai $(\mathrm{N}=241)$

\begin{tabular}{|c|c|c|c|c|c|c|}
\hline & 1 & 2 & 3 & 4 & 5 & 6 \\
\hline $\begin{array}{l}\text { 1. Envolvimento cuidados } \\
\text { diretos }\end{array}$ & & $.44^{* *}$ & $.26^{* *}$ & $34^{* *}$ & $.11^{*}$ & $.15^{*}$ \\
\hline $\begin{array}{l}\text { 2. Envolvimento cuidados } \\
\text { indiretos }\end{array}$ & & & $.26^{* *}$ & $17^{* *}$ & .08 & $.11^{*}$ \\
\hline $\begin{array}{l}\text { 3. Envolvimento ensino/ } \\
\text { disciplina }\end{array}$ & & & & $43^{* *}$ & $.11^{*}$ & $.12^{*}$ \\
\hline $\begin{array}{l}\text { 4. Envolvimento } \\
\text { brincadeira }\end{array}$ & & & & & $.13^{*}$ & $.15^{*}$ \\
\hline 5. Crenças modernas pai & & & & & & $.14^{*}$ \\
\hline 6. Crenças modernas mãe & & & & & & \\
\hline
\end{tabular}

As crenças modernas, de ambos os cuidadores, estão positiva e significativamente associadas com todas as dimensões do envolvimento que implicam interação direta com a criança. Apenas as crenças da mãe estão associadas com o envolvimento nos cuidados indiretos. Existe, ainda, uma associação positiva e significativa das crenças ao nível do casal.

Finalmente, realizaram-se quatro modelos de regressão múltipla hierárquica em que cada dimensão do envolvimento: cuidados diretos, cuidados indiretos, ensino/disciplina e brincadeira foi regredida nas variáveis preditoras agrupadas em três blocos hierárquicos: (a) variáveis sociodemográficas; (b) crenças maternas e paternas sobre o papel do pai, e a interação entre as mesmas; e (c) interação entre crenças e sexo da criança (Tabela 3).

A variância total explicada pelos três blocos de preditores $\left(\Sigma R^{2}\right)$ atingiu significância estatística para os cuidados diretos $(F[13,240]=2.44 ; p=.006)$ e indiretos $(F[13,240]$ $=2.88 ; p=.001)$, contrariamente ao ensino/disciplina $(F[13,240]=1.48 ; p=.135)$ e à brincadeira $(F[13,240]=1.65$; $p=.08)$. Relativamente às variáveis introduzidas nos modelos, verificou-se que para os cuidados diretos o número de horas de trabalho da mãe é preditor de um maior envolvimento relativo do pai, tal como as crenças modernas da mãe, o mesmo não se verificando para as crenças do pai. A interação entre as crenças parentais é marginalmente significativa. Adicionalmente, existe uma interação significativa entre as crenças da mãe e o sexo da criança, sendo que o efeito da crença no envolvimento é maior nas raparigas, do que nos rapazes (Figura 1). De modo a testar os efeitos de interação utilizou-se a analise "simple slopes" (Aiken \& West, 1991; Robinson, Tomek, \& Schumacker, 2013). Decompondo os parâmetros de regressão (slopes) por sexo, o efeito da crença materna no envolvimento relativo do pai em cuidados diretos e nas raparigas é beta $=.25(p<.001)$, enquanto que para os rapazes é beta $=.06$ (n.s.). Um teste de diferenças de coeficiente de correlação " $r$ para $Z$ " indicou que a diferença entre a magnitude dos valores para as raparigas e os rapazes é estatisticamente significativa $(Z=1.91 ; p<.048)$.

Nos cuidados indiretos, a idade e as habilitações académicas dos pais são preditores significativos, ou seja, pais mais novos e com mais habilitações apresentam um maior envolvimento relativo nas atividades de organização/gestão do dia a dia da criança. No ensino/disciplina apenas a variável habilitações académicas do pai tem um efeito significativo. Não se verificaram efeitos significativos no modelo da brincadeira.

\section{Discussão}

Apesar do crescente interesse sobre o papel do pai e do seu impacto no desenvolvimento da criança, do adulto e do próprio sistema familiar, muito há para compreender acerca destes processos e da sua variabilidade em diferentes grupos socioculturais (Cabrera et al., 2018). Os resultados obtidos são indicativos da diversidade em termos das atividades e contextos em que os pais se encontram envolvidos relativamente à mãe, mas também de uma participação tendencialmente baseada no género, potencializando diferentes tipos de interação com a criança. Nas atividades relacionadas com os cuidados é quase sempre a mãe que as realiza, sendo nestes domínios que os pais mais se distanciam de uma participação igualitária, em particular nas tarefas de gestão/organização de rotinas que garantem que necessidades diversas da criança estejam asseguradas (Lamb \& Lewis, 2010). Estas últimas estão associadas com a gestão do domínio familiar e da casa, podendo ser consideradas com menos prazerosas, dado que tendencialmente não implicam interação direta com a criança (e.g., Monteiro et al., 2017; Torres et al., 2014). Assim, e apesar dos dados macrossociais indicarem, que comparativamente com gerações anteriores, o homem português está mais envolvido na vida familiar (Torres et al., 2018; Wall et al., 2016), nesta amostra as diferenças permanecem, com o pai a ter em 
Tabela 3. Parâmetros $B$ estimados e percentagem de variância explicada para os modelos de regressão múltipla hierárquica do envolvimento paterno

\begin{tabular}{|c|c|c|c|c|}
\hline & \multicolumn{4}{|c|}{ Envolvimento relativo do pai } \\
\hline & Cuidados diretos & Cuidados indiretos & Ensinol disciplina & Brincadeira \\
\hline \multicolumn{5}{|l|}{ Bloco 1: Variáveis sociodemográficas } \\
\hline Sexo criança & .04 & -.09 & -.02 & -.06 \\
\hline Idade criança & .11 & -.02 & .06 & .02 \\
\hline Idade mãe & -.03 & .05 & .09 & .13 \\
\hline Idade pai & -.12 & $-.22^{* *}$ & -.11 & -.08 \\
\hline Habilitações mãe & .04 & -.01 & -.09 & .09 \\
\hline Habilitações pai & .11 & $.29^{* *}$ & $.25^{* *}$ & .09 \\
\hline Horas/trabalho mãe & $.23^{* *}$ & .05 & .04 & -.04 \\
\hline Horas/trabalho pai & -.10 & -.11 & -.07 & .02 \\
\hline $\mathrm{R}^{2}$ (variância explicada pelo Bloco 1) & $8.00 \%$ & $13.00 \%$ & $7.90 \%$ & $7.50 \%$ \\
\hline \multicolumn{5}{|l|}{ Bloco 2: Crenças sobre o papel do pai } \\
\hline Crenças modernas pai & -.05 & .07 & .11 & .18 \\
\hline Crenças modernas mãe & $.25^{* *}$ & .14 & .13 & .18 \\
\hline Crenças pai x crenças mãe & $.13^{+}$ & .09 & .11 & .01 \\
\hline$\Delta \mathrm{R}^{2}(\Delta$ variância explicada pelo Bloco 2$)$ & $3.2 \% *$ & $1.00 \%$ & $0.80 \%$ & $1.00 \%$ \\
\hline \multicolumn{5}{|l|}{ Bloco 3: Crenças X sexo criança } \\
\hline Crenças pai $X$ sexo criança & .13 & .02 & -.04 & -.10 \\
\hline Crenças mãe $X$ sexo criança & $-.20^{* *}$ & .11 & -.01 & .11 \\
\hline$\Delta \mathrm{R}^{2}$ ( $\Delta$ variância explicada pelo Bloco 3) & $2.8 \%$ * & $1.00 \%$ & $0.30 \%$ & $0.50 \%$ \\
\hline$\Sigma \mathrm{R}^{2}$ (Variância explicada pelos 3 blocos) & $14 \% * *$ & $15 \%$ ** & $9 \%$ & $9 \%$ \\
\hline
\end{tabular}

${ }^{* *} p<.01 ;{ }^{*} p<.05 ;{ }^{+} p<.10$

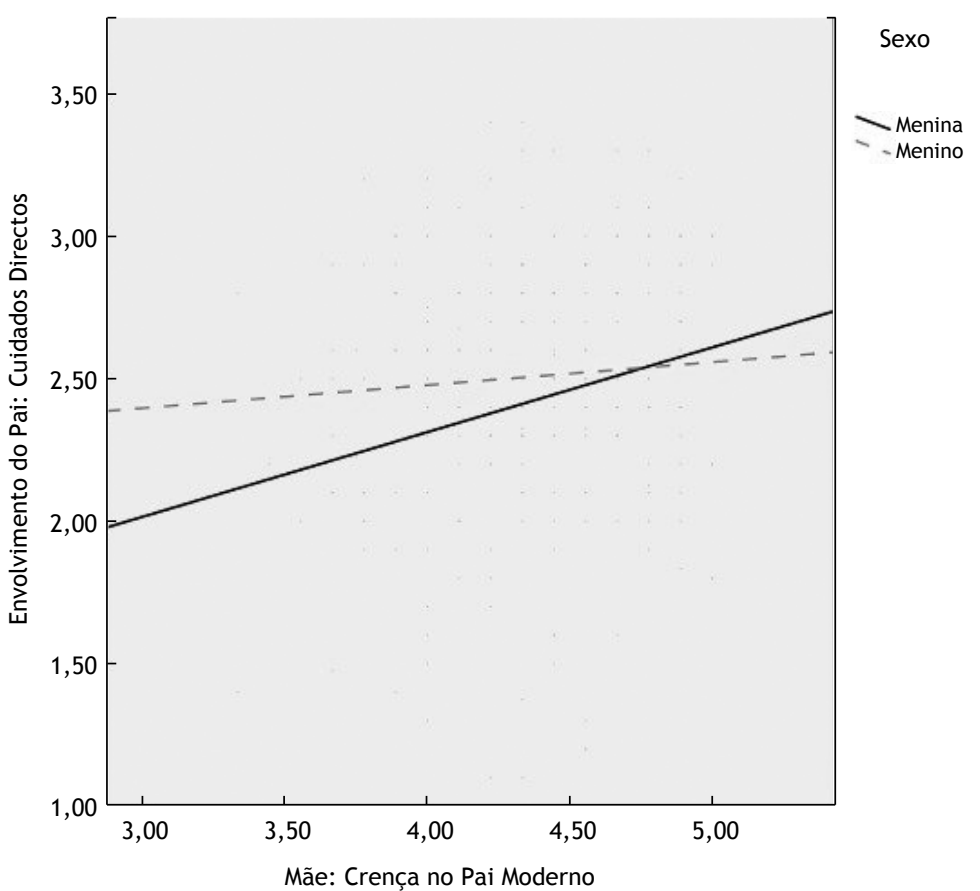

Figura 1. Interação entre crenças maternas e sexo da criança no envolvimento do pai nos cuidados diretos. 
média um papel mais de apoio à mãe, mesmo em famílias de duplo emprego, criando potencialmente situações de desigualdade entre mulheres e homens.

Será, no entanto, fundamental não minimizar o envolvimento do pai noutras atividades, nomeadamente, na brincadeira e no ensino/disciplina onde se verifica uma tendência clara para a participação igualitária, expressando assim a saliência destes contextos (em particular da brincadeira) nas interações pai/criança (Lamb \& Lewis, 2010). Proporcionalmente os pais brincam mais do que cuidam, apoiando a criança na exploração do meio e incentivando-a a assumir riscos sob a sua monitorização (Grossman et al., 2002). Mais recentemente, a investigação tem procurado valorizar e compreender as especificidades dos estilos de brincadeira do pai, e se esta oferece algo de único para o desenvolvimento da criança (StGeorge \& Freeman, 2017).

Uma das possíveis explicações para a variabilidade no envolvimento relativo do pai poderá residir nas suas crenças sobre o papel de pai; outra remeterá para o papel regulador da mãe (via crenças maternas). Nesta amostra, ambos os cuidadores apresentam crenças concordantes com uma visão mais moderna da paternidade, indo ao encontro de estudos na área da sociologia (Wall et al., 2016) que indicam um declínio progressivo, nos últimos anos, na sociedade portuguesa, da crença da mãe como cuidadora natural e com responsabilidades acrescidas na família, mas também que são os homens mais velhos os mais conservadores. Por outro lado, quanto mais horas os pais trabalham mais tradicionais tendem a ser as crenças da mãe, indo no sentido de uma visão de pai como principal provedor financeiro da família. Verificou-se, ainda, que as crenças no casal se encontram significativamente associadas, (Favez et al., 2016), podendo potencializar maior cooperação entre os cuidadores.

Embora, ao nível das associações bivariadas, se verifique que quanto mais progressistas são as crenças dos pais, mais estes estão envolvidos em atividades que implicam interação direta com a criança (e.g., Bornstein, 2002), tal não se verifica ao nível dos modelos testados para nenhum tipo de atividade. Também as crenças da mãe se encontram associadas com um maior envolvimento do pai (inclusivamente nos cuidados indiretos), no entanto, apenas preveem o envolvimento nos cuidados diretos. Alguns autores salientam a existência de discrepâncias entre as expetativas socioculturais, as crenças sobre o papel do pai e os comportamentos parentais ao nível do que estes fazem no dia a dia com a criança. Tal poderá ser gerador de stress e de menor bemestar nos adultos e na família (e.g., Milkie, Bianchi, Mattingly, \& Robinson, 2002), sendo este um ponto a explorar em estudos futuros.

Relativamente ao trabalho das figuras parentais, apenas as horas de trabalho da mãe são um preditor significativo da participação do pai nos cuidados diretos, indo ao encontro da noção de que, em famílias de duplo-emprego, um maior envolvimento do pai seria atribuído (em parte) à pressão criada pelo trabalho materno. Nestas famílias há que encontrar um equilíbrio entre as esferas profissional e familiar, em particular, no que se refere aos cuidados da criança, sendo uma maior contribuição do homem neste domínio vista como um passo nesse sentido (e.g., Lamb, 2010; Wall et al., 2016).

Estes resultados apontam, ainda, para a importância de se considerar a função reguladora (gatekeeping) da mãe, em particular, no domínio dos cuidados diretos (Allen \&
Hawkins, 1999; McBride et al., 2005; Schoppe-Sullivan et al., 2008). Esta foi, ainda, explorada ao analisar-se a interação entre crenças maternas e sexo da criança, verificando-se que o efeito é maior para as raparigas, do que para os rapazes. Nesta amostra, o sexo dos cuidadores (mãe) e da criança (feminino) parece, assim, ter impacto no tipo de interações ocorridas. Coloca-se a hipótese de que, face a atividades como o dar banho ou vestir, com crianças já em idade pré-escolar, as mães considerem que devem ser elas a realizá-las com as filhas, revertendo para uma visão mais tradicional dos papeis parentais. Em sociedades que promovem a igualdade de género os estereótipos são muitas vezes implícitos e inconscientes, emergindo de formar menos clara (e.g., Mesman \& Groeneveld, 2017).

Relativamente aos cuidados indiretos os únicos preditores significativos foram a idade e as habilitações académicas do pai, com estas últimas a preverem também o ensino/ disciplina (e.g., Monteiro et al., 2017). É possível que cuidadores mais novos e com maiores habilitações tenham atitudes mais progressistas e igualitárias sobre os papeis de género (para além do que é ser pai/mãe), promovendo assim o assumir de maior responsabilidade no contexto familiar (Jacobs \& Kelley, 2006). As dimensões do ensino/disciplina e da brincadeira são dois domínios tradicionalmente associados aos pais (e.g., Lamb \& Lewis, 2010, Parke, 1996, 2000), e onde tendencialmente partilham atividades com as mães, o que poderá ajudar a explicar que as crenças do pai, assim como as da mãe não sejam preditores significativos do seu envolvimento.

Os autores gostariam de indicar algumas limitações do estudo, nomeadamente, o facto de ter um delineamento transversal e de utilizar apenas medidas de autorrelato, com a possibilidade de algum enviesamento social, nomeadamente, no que concerne à medida das crenças. Considerando as novas expectativas face aos papeis de género é possível que os cuidadores sejam relutantes em expressar crenças e visões mais tradicionais, pelo que será importante a utilização de metodologias de observação em estudos futuros. Os resultados obtidos têm, ainda, que ser enquadrados nas características desta amostra, que é de conveniência, e no contexto de outras potenciais variáveis explicativas deste fenómeno. As atitudes e crenças parentais podem ter diferentes significados e consequências para o envolvimento em função das diversas tipologias familiares, da origem étnica ou social dos sujeitos, ou do seu baixo estatuto socioeconómico. Será expectável que fatores económicos influenciem a capacidade e oportunidade dos pais estarem ativa e positivamente envolvidos com os seus filhos (e.g., baixo nível de escolaridade, horários menos flexíveis e longos de trabalho, ausência de ou reduzidas redes de suporte social), pelo que estas variáveis devem ser incluídas em estudos futuros, potencializando a adequação dos programas parentais a um público diverso.

A análise das crenças sobre os papéis parentais e de género poderá ser um importante contributo para a compreensão da ambivalência/resitência que mães e pais possam ter face a um maior envolvimento do homem no dia a dia da criança, e em atividades específicas; potencializando assim um trabalho colaborativo e positivo entre os cuidadores (Allen \& Hawkins, 1999), ou entre família e outros contextos como a escola (e.g., Instituto del Niño y Adolescente del Uruguay, 2017). 


\section{Referências}

Afonso, A., Veríssimo, M., Fernandes, M., Borges, P., \& Monteiro, L. (2011). Associações entre o envolvimento paterno e a competência social de crianças em contexto pré-escolar. Psicologia Educação Cultura, 15(1), 43-60.

Aiken, L. S., \& West, S. G. (1991). Multiple regression: Testing and interpreting interactions. Thousand Oaks, CA: Sage Publications.

Allen, S., \& Hawkins, A. (1999). Maternal gatekeeping: Mothers' beliefs and behaviors that inhibit greater father involvement in family work. Journal of Marriage and Family, 61(1), 199-212. http://dx.doi.org/10.2307/353894

Bailey, W. T. (1994). A longitudinal study of father's involvement with young children: Infancy to age 5 years old. Journal of Genetic Psychology, 155, 331-339. http://dx.doi.org/10.1080/0 0221325.1994.9914783

Beitel, A., \& Parke, R. D. (1998). Maternal and paternal attitudes as determinants of father involvement. Journal of Family Psychology, 12, 268-288.

Buckley, C. K., \& Schoppe-Sullivan, S. J. (2010). Father involvement and coparenting behavior: Parents' nontraditional beliefs and family earner status as moderators. Personal Relationships, 17, 413-431.

Bornstein, M. H. (2002). Parenting infants. Em M. H. Bornstein (Ed.), Handbook of parenting: Children and parenting (pp. 3-43). Mahwah, NJ: Lawrence Erlbaum Associates Publishers.

Cabrera, N. J., Volling, B. L., \& Barr, R. (2018). Fathers are parents, too! Widening the lens on parenting for children's. Child Development Perspectives, 12(3), 152-157. http://dx.doi. org/10.1111/cdep.12275

Cabrera, N. J., Tamis-LeMonda, C. S., Bradley, R. H., Hofferth, S., \& Lamb, M. E. (2000). Fatherhood in the twenty-first century. Child Development, 71(1), 127-136. http://dx.doi. org/10.1111/1467-8624.00126

Fagan, J., \& Barnett, M. (2003). The relationship between maternal gatekeeping, paternal competence, mothers' attitudes about the father role, and father involvement. Journal of Family Issues, 24(8), 1020-1043. http://dx.doi.org/10.1177/0192513X03256397

Favez, N., Tissot, H., Frascarolo, F., Stiefel, F., \& Despland, J. N. (2016). Sense of competence and beliefs about parental roles in mothers and fathers as predictors of coparenting and child engagement in mother-father-infant triadic interactions. Infant and Child Development, 25(4), 283-301. http://dx.doi. org/10.1002/icd.1934

Freeman, H., Newland, L. A., \& Coyl, D. D. (2008). Fathers beliefs as a mediator between contextual barriers and father involvement. Early Child Development and Care, 178, 7-8, 803-819. http://dx.doi.org/10.1080/03004430802352228

Fuertes, M., Faria, A., Beeghly, M., \& Lopes-dos-Santos, P. (2016). The effects of parental sensitivity and involvement in caregiving on mother-infant and father-infant attachment in a Portuguese sample. Journal of Family Psychology, 30, 147-156. http://dx.doi.org/10.1037/fam0000139

Grossmann, K., Grossmann, K. E., Fremmer-Bombik, E., Kindler, H., Scheuerer-Englisch, H., \& Zimmermann, P. (2002). The uniqueness of the child-father attachment relationship: fathers' sensitive and challenging play as a pivotal variable in a 16 -year longitudinal study. Social Development, 11(3), 301-3317. http:// dx.doi.org/10.1111/1467-9507.00202

Instituto del Niño y Adolescente del Uruguay. (2017). Incidencia de los centros de atención a la primera infancia de INAU en el ejercicio de las parentalidades. Montevideo, Uruguay: INAU. Disponível em https://www.plancaif.org.uy/documentos/equipos-de-trabajo/item/download/149_3a013569886e7ffb343d22eaf1667d91

Jacobs, J. N., \& Kelley, M. L. (2006). Predictors of paternal involvement in childcare with dual-earner families with young children. Fathering, 4(1), 23-47. http://dx.doi.org/10.3149/ fth.0401.23
Lamb, M. E. (2010). How do fathers influence children's development? Let me count the ways. In M. E. Lamb (Ed.), The role of the father in child development (pp.1-26). Hoboken, NJ: John Wiley \& Sons, Inc.

Lamb, M. E., \& Tamis-LeMonda, C. S. (2004). The role of the father: An introduction. En M. E. Lamb (Ed.), The role of the father in child development (pp.1-31). Hoboken, NJ: John Wiley \& Sons, Inc.

Lamb, M. E., \& Lewis, C. (2010). The development and significance of father-child relationships in two-parent families. Em M. E. Lamb (Ed.), The role of the father in child development (pp. 94-153). Hoboken, NJ: John Wiley \& Sons, Inc.

Lima, J. (2005). O envolvimento paterno nos processos de socialização da criança. Em J. B. Ruivo (Ed.), Desenvolvimento: Contextos familiares e educativos (pp. 200-233). Porto, Portugal: Livpsic.

Marsiglio, W., Amato, P., Day, R. D., \& Lamb, M. E. (2000). Scholarship on fatherhood in the 1990s and beyond. Journal of Marriage and the family, 62(4), 1173-1191. http://dx.doi.org/10.1111/ j.1741-3737.2000.01173.x

Mesman, J., \& Groeneveld, M. G. (2017). Gendered parenting in early childhood: Subtle but unmistakable if you know where to look. Child Development Perspectives, 12(1), 22-27. http:// dx.doi.org/10.1111/cdep.12250

Mikelson, K. S. (2008). He said, she said: Comparing mother and father reports of father involvement. Journal of Marriage and Family, 70(3), 613-624. http://dx.doi.org/10.1111/j. 1741-3737.2008.00509.x

Milkie, M. A., Bianchi S. M., Mattingly, M. J., \& Robinson, J. P. (2002). Gendered division of childrearing: Ideals, realities, and the relationship to parental well-being. Sex Roles, 47(1-2), 2138. http://dx.doi.org/10.1023/A:1020627602889

McBride, B. A., Brown, G. L., Bost, K. K., Shin, N., Vaughn, B., \& Korth, B. (2005). Paternal identity, maternal gatekeeping, and father involvement. Family Relations, 54(3), 360-372. http:// dx.doi.org/10.1111/j.1741-3729.2005.00323.x

Monteiro, L., Fernandes, M., Veríssimo, M., Pessoa e Costa, I., Torres, N., \& Vaughn, B. E. (2010). Perspetiva do pai acerca do seu envolvimento em famílias nucleares. Associações com o que é desejado pela mãe e com as características da criança. Revista Interamericana de Psicologia, 44(1), 120-130.

Monteiro, L., Fernandes, M., Torres, C., Santos, C. (2017). Father's involvement and parenting styles in Portuguese families. The role of education and working hours. Análise Psicológica, 4 (XXXV): 513-528. http://dx.doi.org/10.14417/ap.1451

Monteiro, L., Veríssimo, M., \& Pessoa e Costa, I. (2008). Escala de Envolvimento Parental: Actividades de cuidados e de socialização (Unpublished manual). Lisboa, Portugal: ISPA - Instituto Universitário.

Monteiro, L., Torres, N. Branco, I., Pessoa-e-Costa, I., \& Santos, C. (2015). Father involvement in care and socialization activities with children: Is it all about fathers or are mothers being gatekeepers? Poster apresentado no SRCD Biennial Meeting, Philadelphia, Pennsylvania, USA.

NICHD Early Child Care Research Network. (2000). Factors associated with fathers' caregiving activities and sensitivity with young children. Journal of Family Psychology, 14(2), 200-219. http://dx.doi.org/10.1037//D893-3200.14.2.200

Palkovitz, R. (2002). Involved fathering and men's adult development. Mahwa: Lawrence Erlbaum Associates, Inc.

Parke, R. D. (2000). Father involvement. A developmental psychological perspective. Marriage \& Family Review, 29(2-3), 43-58. http://dx.doi.org/10.1300/J002v29n02_04

Parke, R. D. (1996). Fatherhood. Cambridge, MA: Harvard University Press.

Pleck, J. H., \& Masciadrelli, B. P. (2004). Paternal involvement by U.S. residential fathers: Levels, sources, and consequences. $\mathrm{Em} \mathrm{M.} \mathrm{E.} \mathrm{Lamb} \mathrm{(Ed.),} \mathrm{The} \mathrm{role} \mathrm{of} \mathrm{the} \mathrm{father} \mathrm{in} \mathrm{child} \mathrm{develop-}$ ment (pp. 222-271). Hoboken, NJ: John Wiley \& Sons Inc. 
Robinson, C. D., Tomek, S. \& Schumacker, R. E. (2013) Tests of moderation effects: Difference in simple slopes versus the interaction term. Multiple Linear Regression Viewpoints, 39(1), 16-24.

Schoppe, S. J. (2001). What is a father? Unpublished manuscript, University of Illinois at Urbana-Champaign.

Schoppe-Sullivan, S. J., Brown, G. L., Cannon, E. A., Mangelsdorf, S. C., \& Sokolowski, M. S. (2008). Maternal gatekeeping, coparenting quality, and fathering behavior in families with infants. Journal of Family Psychology, 22(3), 389-398. http:// dx.doi.org/10.1037/0893-3200.22.3.389

Stgeorge, J., \& Freeman, E. (2017). Measurement of father-child rough-and-tumble play and its relations to child behavior. Infant Mental Health Journal, 38(6), 709-725. http://dx.doi. org/10.1002/imhj.21676
Torres, A., Pinto, P. C., Costa, D., Coelho, B., Maciel, D., Reigadinha, T., \& Theodoro, E. (2018). Igualdade de género ao longo da vida: Portugal no contexto europeu. Lisboa, Portugal: Guide-Artes Gráficas, Lda.

Torres, N., Veríssimo, M., Monteiro, L., Ribeiro, O., \& Santos, A. J. (2014). Domains of father involvement, social competence and problem behavior in preschool children. Journal of Family Studies, 20(3), 188-203. http://dx.doi.org/10.1080/13229400.2 014.11082006

Wall, K., Cunha, V., Atalaia, S., Rodrigues, L., Correia, R., Correia, S. V., \& Rosa, R. (2016). Livro branco. Homens e igualdade de género em Portugal. Lisboa, Portugal: Editorial do Ministério da Educação e Ciência. 